\title{
Node Selection for Cooperative Localization: Efficient Energy vs. Accuracy Trade-off
}

\author{
Albert Bel ${ }^{1}$, José López Vicario ${ }^{2}$, Gonzalo Seco-Granados ${ }^{3}$ \\ Group of Signal Processing for Communication and Navigation (SPCOMNAV) \\ Telecommunications and Systems Engineering Department, Universitat Autonoma de Barcelona (UAB) \\ Edifici $Q$ - Campus de la UAB, 08193 Bellaterra (Barcelona), Spain \\ Phone: +34 9358138 43, Fax +34935814031 \\ 1 albert.bel@uab.cat \\ 2 jose.vicario@uab.cat \\ 3 gonzalo.seco@uab.cat
}

\begin{abstract}
In this paper, we present a cooperative localization algorithm for wireless sensor networks. We adopt an RSS-based localization algorithm due to its lower complexity with respect to other ranging techniques such as TOA or AOA. Since the energy consumption is a key aspect in sensor networks, we propose a node selection algorithm in order to avoid the use of all the nodes in the network for positioning purposes. Thus, we derive a practical solution tailored to the strict requirements of sensor networks in terms of complexity, size and cost. In particular, nodes in the network select the cooperating nodes by simply comparing their received RSS with a threshold. We present results based on both computer simulations and experimentation with the Crossbow IRIS motes showing that the proposed scheme offers a good trade-off in terms of position accuracy vs. energy consumption.
\end{abstract}

\section{INTRODUCTION}

In recent years, location estimation in wireless sensor networks (WSN) has raised a lot of interest from researchers. The idea of having sensing data whithout any information of the location does not make sense. Existing techniques, such as GPS, are, usually, inappropriate in large scale networks, due to the increase in the cost and size of the nodes. For that reason a variety of location estimation techniques has been proposed for wireless sensor networks [1].

Existing algorithms could be classified as: centralized versus distributed, and non-cooperative versus cooperative. In centralized algorithms, it is a central processor that receives all the information and calculates the position of all the network nodes. This central processor will probably not have any processing limitation, but on the other hand, this solution limits the scalability of the system. Hence, centralized solutions are not very attractive for large scale networks, where distributed algorithms are prefered.

The second classification differentiates between the cooperative and non-cooperative techniques [2]. We assume that a network is formed by nodes with known position, called reference or anchor nodes, and nodes without knowing their position, called non-located nodes. In non-cooperative techniques, non-located nodes are only able to communicate with reference nodes. On the other hand, cooperative techniques al- low an non-located node to communicate with any node either anchor or non-located. In general, cooperative techniques can increase localization performance in terms of both accuracy and coverage.

Typically, all these methods consist in two phases. The first phase is the measurement phase, in which nodes estimate signal metrics based on direct communications with the other nodes. And the second phase is the location-update phase, in which the nodes modify their own state using the estimated signal metrics and state information of the rest of the nodes.

The most common techniques used in the measurement phase are: received signal strength (RSS), time-of-arrival (TOA), and angle-of-arrival (AOA) [3], [4]. With these techniques, relative distances between nodes are estimated by means of a packet exchange between nodes. These range measurements will be affected by different sources of error that will cause uncertainty at the distance estimation. In the case of TOA-based [5] or AOA-based systems [6], one could obtain an accurate estimation, but complexity is increased due to the necessity of specific protocols (TOA) or additional hardware requirements (AOA).

RSS-based estimations are based on the well-known radio propagation pathloss model. This technique has become the most inexpensive and simple one because RSS or RF signals can be measured during normal transmissions between nodes. The major sources of error are shadowing and multipath signals, that complicates the a priori modeling of the channel. Indeed, the error introduced in the measurements is multiplicative with the distance [3]. This behavior introduces a problem in cooperative algorithms. Basically, distance estimates are degraded when the cooperation between distant nodes is allowed [7].

In this paper, we focus on cooperative localization based on RSS. In order to reduce the effect of RSS impairments, we propose a node selection algorithm aimed at selecting only those nodes providing valuable information (in terms of RSS measurements quality). Furthermore, the node selection strategy is also devoted to limit the cooperation mechanisms in order to minimize energy consumption. More specifically, the 


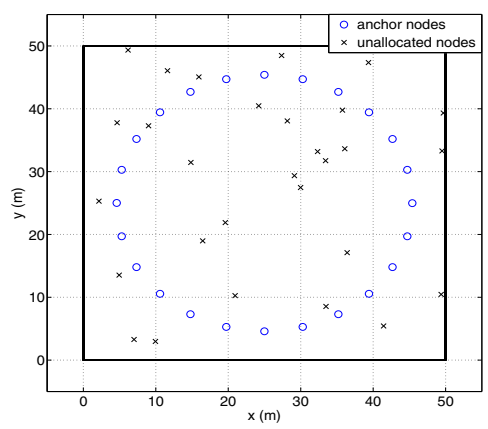

Fig. 1. Example of an scenario deployment

proposed algorithm is aimed at optimizing the existing tradeoff in terms of position accuracy versus energy consumption.

\section{System MOdeL}

Let us consider a network with $N$ nodes. There are $N_{1}$ nodes, whose exact locations are known (anchor nodes). The location of the rest of nodes $\left(N_{2}=N-N_{1}\right)$ is unknown (nonlocated nodes). The main goal is to estimate the location of the non-located nodes with the help of anchor nodes. Concerning the anchor nodes placement, we follow the approach presented in [8], where it is shown that the best anchor placement is a centered circumference of radius equal to the root-meansquare (RMS) of the non-located nodes distances to the center. One can see in Figure 1 an example of the scenario deployment.

\section{A. Measurement phase}

As commented previously, we will consider an RSS-based distributed cooperative algorithm for location estimation. The first phase of the algorithm consists in obtaining internode distances through RSS measurements. Distance will be obtained through the classical radio-propagation pathloss and shadowing model [7]. Received Signal Strength (RSS) can be expressed as the power received in node $i$ from a signal transmitted by node $j, P_{i j}$, as:

$$
R S S_{i j}=P_{i j}=P_{0}-10 \alpha \log _{10} d_{i j}-v_{i j}(\mathrm{dBm})
$$

where $P_{0}$ is the power received in $\mathrm{dBm}$ at $1 \mathrm{~m}$ distance, $d_{i j}$ is the distance between nodes $i$ and $j$, parameter $\alpha$ is the pathloss exponent and $v_{i j} \sim N\left(0, \sigma_{v}^{2}\right)$ represents log-normal shadowfading effects, where the value of the standard deviation $\sigma_{v}$ depends on the characteristics of the environment. Given the power received $R S S_{i j}$ in (1), the MLE of the distance $d_{i j}$ between node $i$ and $j$ is derived. As a result, an estimate of the actual distance is obtained, which is referred as $\delta_{i j}$.

\section{B. LS Cooperative algorithm}

Once the relative distances between nodes have been obtained, the position estimates for each non-located node are obtained by means of the Least-Squares criterion. Position estimates are calculated by obtaining the set of non-located node positions that minimize the Euclidean distance between measured distances at the first phase and the distances computed using such position estimates. This is reflected in the following expression:

$$
C_{L S}(\mathbf{x})=\sum_{i=1}^{N_{2}} \sum_{j \in S_{i}}\left\|\delta_{i j}-\hat{d}_{i j}\right\|^{2}
$$

where $\hat{d}_{i j}$ is the distance between nodes $i$ and $j$, calculated with the estimated position (or real coordinates if node $j$ is an anchor) of nodes $i$ and $j, S_{i}$ is the number of nodes (anchor and non-located) that cooperates in the position estimation of non-located node $i$, and $\mathbf{x}$ are the coordinates of nodes.

The minimization of the cost function of (2) will carried out with a distributed iterative method. We adopt a distributed strategy due to its scalability and robustness. Each nonlocated node iteratively computes, at each time instant $t$, its own coordinates $\hat{\mathbf{x}}_{\mathbf{i}}$ by minimizing $C_{L S}$ through a distributed gradient descent mechanism:

$$
\hat{\mathbf{x}}_{i}(t)=\hat{\mathbf{x}}_{i}(t-1)+\gamma \sum_{j \in S_{i}}\left(\delta_{i j}-\hat{d}_{i j}\right) \mathbf{e}_{\mathbf{i j}}
$$

where $\mathbf{e}_{\mathbf{i j}}$ is the unit vector that takes the orientation between the node $i$ and node $j$ and $\gamma$ is the step size.

As iterative methods depend on initial values, in the literature, are presented many methods to obtain better initial values for the variables. In that sense, is presented in [7], the concept of collaborative methods, in which the initial values are obtained with a proper localization algorithm, e.g MultiDimensional Scaling (MDS). It is a good option but it increases the computational cost. Another proposal is the initialization with random values. It is a simple method but it would result in a higher number of iterations. We propose to intialize each $\hat{\mathbf{x}}_{\mathbf{i}}(0)$ with the coordinates of the nearest anchor node. When the node $i$ set its own group $S_{i}$, it has to find the nearest anchor node and take its coordinates as its own initial coordinates. In the case that an anchor node is not present, random coordinates are selected. By doing so, an initial value closer to the final solution is obtained without increasing the computational cost.

\section{Energy consumption model}

In this paper we also control the energy consumption of the network. To do so, we consider the model of the total amount of energy consumed presented in [9]:

$$
\epsilon=\left(\mu_{R_{x}}+\mu_{T_{x}}\right)\left(\sum_{i=1}^{N_{2}} S_{i}-N_{2}\right) \kappa
$$

where $\kappa$ is the number of iterations of the algorithm and $\mu_{T_{x}}$ and $\mu_{R_{x}}$ are the energy consumption to transmit and receive, respectively, a message. 


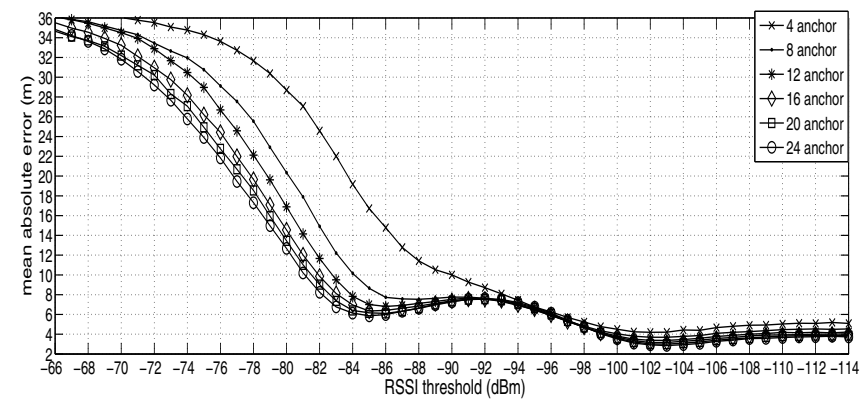

Fig. 2. Mean absolute error versus RSS threshold

\section{Selection Mechanism}

As commented above, this paper is focused on the derivation of a node selection algorithm in order to restrict the cooperation mechanism to those nodes providing accurate RSS measurements. Next, we present the derivation process of such algorithm.

\section{A. Motivation}

Consider a cooperative scenario where one node is only allowed to cooperate with those nodes having an RSS higher than a specific threshold $R S S_{t h}$ (i.e., node $j$ cooperates with node $i$ if $R S S_{i j}>R S S_{t h}$ ). In Figure 2, we present the mean absolute error obtained in the position estimates of such a scenario as a function of the selected threshold. It is observed that lowers errors can be obtained with lower thresholds, i.e. by allowing the cooperation with a higher number of nodes. However, it is remarkable that the error is not monotically decreasing and the error is saturated for low thresholds values. This is basically due to the effects commented previously that having a further node becomes in a higher error in distance estimate. On the other hand exists, also a relation with the presence of more anchor nodes cooperating in the estimation of coordinates. For that reason, we propose to limit the number of cooperating nodes by selecting a threshold value that provides an appropiate error value in terms of position accuracy. The use of a selection threshold is aligned with the WSN philosophy in terms of reduced complexity, size and cost of the terminals. By doing so, the number of cooperating nodes, those within group $S_{i}$, is reduced and energy efficiency can be improved. For example, the difference in error obtained with a threshold of $-88 \mathrm{dBm}$ or $-95 \mathrm{dBm}$ is minimal but the number of cooperating nodes can be further reduced with the first option, i.e. thus reducing energy consumption.

\section{B. Selection Criterion}

In order to select an appropiate RSS threshold, the use of an exhaustive search based on computer simulations is mandatory, due to the complexity involved in the problem at hand. The procedure is even more complex when the large variety of possible scenarios is taken into consideration. In order to avoid that, we propose a simpler method. Basically, the idea is to select the highest RSS threshold that assures that a minimum number of anchor nodes are cooperating with the considered

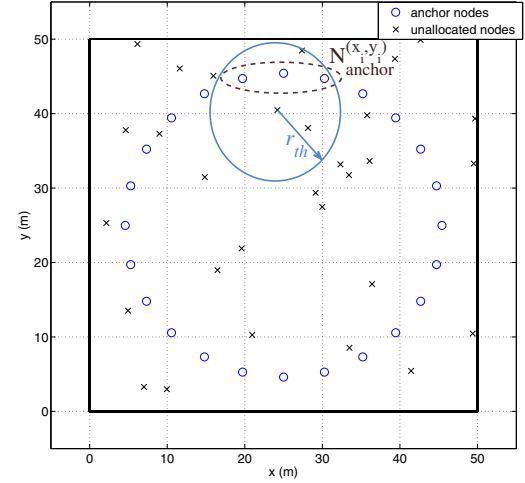

Fig. 3. Number of anchor nodes inside the radio range of a node

non-located node. Clearly this criterion also implies that a set of non-located nodes will be also present in the region of interest for cooperation purposes. In particular, we first fix the mean number of anchor nodes and using this number the RSS threshold is obtained. This metodology provides us a closed-form expression to obtain the required threshold for each possible scenario. Next, we present how such closedform expressions can be obtained.

The number of anchor nodes inside the range of node $i$ is (see Figure 3):

$$
N_{\text {anchor }}^{\left(x_{i}, y_{i}\right)}=\sum_{j=1}^{N_{1}} I\left(\left\|\left(x_{i}, y_{i}\right)-\left(x_{j}^{a}, y_{j}^{a}\right)\right\|<r_{t h}\right)
$$

where $I()$ is the indicator function (i.e. $I(a)=1$ if a is true) and $\left(x_{i}, y_{i}\right)$ and $\left(x_{j}^{a}, y_{j}^{a}\right)$ are the coordinates of the non-located node $i$ and anchor node $j$, respectively.

Then, the mean number of anchor nodes inside the range of node $i$ is:

$$
N_{m_{i}}=\int_{x} \int_{y} N_{\text {anchor }}^{\left(x_{i}, y_{i}\right)} f\left(x_{i}, y_{i}\right) d x_{i} d y_{i}
$$

where $f(x, y)$ is the p.d.f of the nodes position distribution along the area. Without loss of generality, we consider a uniform distribution of the non-located nodes in the network (for a given time realization). Introducing (5) in (6) we obtain:

$$
N_{m}=\int_{x} \int_{y} \sum_{j=1}^{N_{1}} I\left(\left\|\left(x_{i}, y_{i}\right)-\left(x_{j}, y_{j}\right)\right\|<r_{t h}\right) \frac{1}{A} d x_{i} d y_{i}
$$

where $A$ is the total area of the considered scenario. Since the nodes are uniformly distributed the mean number of anchor nodes inside a radius $r_{t h}$ becomes:

$$
N_{m} \approx \sum_{j=1}^{N_{1}} \pi r_{t h}^{2} \frac{1}{A}=\frac{N_{1} \pi r_{t h}^{2}}{A}
$$

Once we have the closed-form expression in (8), we need to establish the dependency of the coverage range radius with the RSS threshold. We will consider two different approximations based on different propagation models. 


\section{- Pathloss model}

In this case we only consider the pathloss model dependency. In other words, we model the received power as a function inversely proportional to the distance elevated to the pathloss exponent.

$$
R S S^{P L}=\frac{P_{0}}{r^{\alpha}}
$$

If the expression of $r_{t h}$ obtained from (8) is introduced in (9), the RSS threshold becomes:

$$
R S S_{t h}^{P L}=\left(\frac{N_{1} \pi P_{0}^{2 / \alpha}}{N_{m} A}\right)^{\alpha / 2}
$$

- Pathloss \& shadowing model

In this case, we consider both the effects of pathloss and shadowing, appearing in (1). The relation between radio range radius and received power is expressed as follows:

$$
R S S^{P L \& S}=\frac{P_{0}}{r^{\alpha}} z
$$

being $z$ a log-normal variable, $z=10^{v / 10} \sim \log \mathcal{N}\left(0, \sigma_{v}^{2}\right)$. Therefore, $N_{m}$ is also a random variable in this case. Taking this into account, we need to search the mean value of $N_{m}$. Using the value of the $r_{t h}$ in (8) and being $E[Z]=e^{\mu_{v}+\frac{1}{2} \sigma_{v}^{2}}$ the mean of a lognormal variable, we can write:

$$
\begin{aligned}
\bar{N}_{m}=E\left[N_{m}\right] & =\frac{\pi N_{1}}{A}\left(\frac{P_{0}}{R S S_{t h}^{P L \& S}}\right)^{2 / \alpha} E\left[z^{2 / \alpha}\right] \\
& =\frac{\pi N_{1}}{A} \frac{P_{0}^{2 / \alpha}}{\left(R S S_{t h}^{P L \& S}\right)^{2 / \alpha}} e^{\frac{1}{2} \frac{4 \sigma_{v}^{2}(\ln 10)^{2}}{\alpha^{2} 100}}
\end{aligned}
$$

As in the previous case, this dependency is combined with eq. (8) and the result is the following RSS threshold:

$$
R S S_{t h}^{P L \& S}=\left(\frac{N_{1} \pi P_{0}^{2 / \alpha} e^{\frac{\sigma_{v}^{2} 2(\log 10)^{2}}{100 \alpha^{2}}}}{\bar{N}_{m} A}\right)^{\alpha / 2}
$$

As observed, eqs. (10) and (13) allow us the computation of the required threshold in terms of the desired mean number of anchor nodes $\left(N_{m}\right)$.

In order to show the validity of the obtained expressions, we compare in Figure 4 such approximations with the actual dependency of $N_{m}$ with the RSS threshold obtained by means of computer simulations. The three different thresholds shown are: $R S S_{t h}^{s i m}$, which is the RSS threshold obtained with simulations that assures a number $N_{m}$ of anchor nodes; $R S S_{t h}^{P L}$, which is the RSS threshold obtained with (10) (pathloss model); and $R S S_{t h}^{P L \& S}$, which is the RSS threshold obtained with . (13) (pathloss \& shadowing model). As observed in Figure 4, both equations achieve a good aproximation when high values of RSS threshold are considered. For low values of RSS threshold the behavior of both approximations becomes worse. This is because the $N_{m}$ value of the eq. (6) is obtained by disregarding the edges of the area (see Figure 5). Therefore, as the RSS threshold is reduced, the coverage radius increases and an extra area outside the edges is considered. As observed

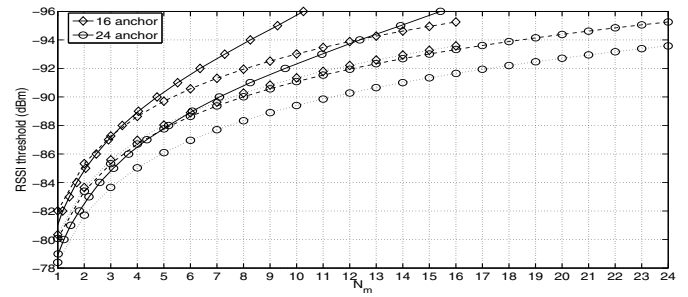

Fig. 4. RSS threshold versus mean number of anchor nodes ((solid line $R S S_{t h}^{s i m}$, dashed line $R S S_{t h}^{P L}$, dashed-dotted line $\left.\left.R S S_{t h}^{P L \& S}\right)\right)$

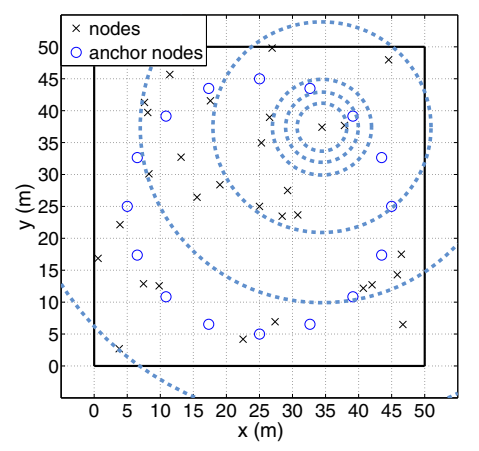

Fig. 5. Effect of increasing the RSS threshold

in the following, this effect has not a big impact on our design as we focus on selecting only the closest nodes.

Besides, Figure 4 shows that the best approximation is obtained with the pathloss model (eq. (10)) and for that reason we focus on this scheme in the sequel. The problem with the approximation given by (13) is that the shadowing effects overestimate the RSS when the power is expressed in lineal (as adopted in (13)).

\section{NUMERICAL RESULTS}

This section presents the performance of the selection criterion. Simulations have been done simulating an indoor scenario. RSS-based systems introduce a great technical difficulty to develop a location system in indoor scenarios. This is because, relationship between the RSS values and the distance estimations depends, to a great extent, on the propagation environment. For that reason, we focus on this challenging scenario in order to show the value of the proposed strategy for wireless sensor networks adopting RSS-based localization. The experimental and simulation parameters are shown in Table I. The minimum $R S S_{\text {treshold }}(-96 \mathrm{dBm})$ is the sensitivity of the IRIS motes [10] used in the experimental results. Hence we consider this as the minimum achievable threshold.

\section{A. Computer simulation}

In Figure 6, it is plotted the mean absolute error for different values of $N_{m}$. The results show that in a 24 anchor scenario the error is $\approx 5.9 \mathrm{~m}$ with a value of $N_{m}$ equal to 3 . If we compare with the error at maximum radio range, equivalent to $N_{m}=15$, the difference in terms of error is approximately 
TABLE I

SiMUlation PARAMETERS

\begin{tabular}{lc}
\hline \hline Simulation Parameters & Parameter Value \\
\hline \hline Size of sensor field & $50 \mathrm{~m} \mathrm{x} \mathrm{50} \mathrm{\textrm {m }}$ \\
Number of non-located nodes $\left(N_{2}\right)$ & 30 \\
Distance-power gradient $\alpha$ & 2.2 \\
Standard deviation $\sigma_{v}$ & $4 \mathrm{~dB}$ \\
First-meter RSS $P_{0}$ & $-63.2 \mathrm{dBm}$ \\
Minimum $R S S_{t h}$ (IRIS hardware constraint) & $-96 \mathrm{dBm}$ \\
Anchor radius & $20.4 \mathrm{~m}$ \\
energy consumption to transmit or receive $\mu_{T_{x}}$ or $\mu_{R_{x}}$ & $400 \mathrm{~nJ}$ \\
\hline
\end{tabular}

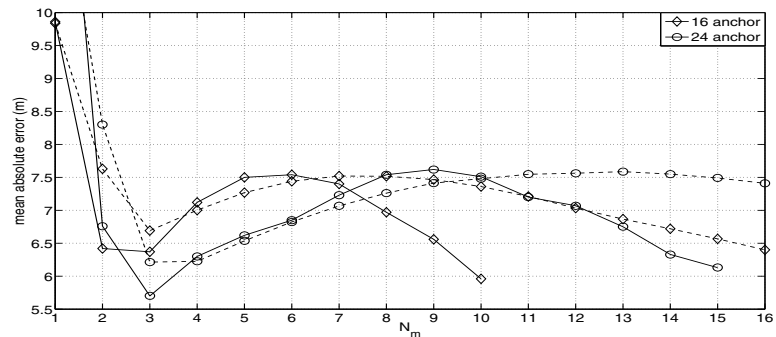

Fig. 6. Mean absolute error versus mean number of anchor nodes(solid line: $R S S_{\text {th }}^{\text {sim }}$, dashed line: $R S S_{t h}^{P L}$ )

of $\approx 0.23 \mathrm{~m}$. This maximum range is fixed by the sensitivity of the receivers. In the 24 anchor scenario, we obtain a better performance with a lower value of $N_{m}$. If we observe the 16 anchor node scenario, the mean absolute error value at the maximum radio range, corresponding to $N_{m}=10$, is $\approx$ $5.96 \mathrm{~m}$. If we set the value of $N_{m}$ equal to 3 , we achieved an error of $\approx 6.4 \mathrm{~m}$. We obtain an increment in the error of $\approx 0.44 \mathrm{~m}$, with respect to the error results at the maximum range.

One can see that at the maximum range, both scenarios (16 anchor and 24 anchor) achieve the same error performance. In that case, the 16 anchor scenario needs a lower value of $N_{m}$. It is a normal behavior because the RSS threshold needed to achieve a certain $N_{m}$ is higher in a 16 anchor scenario. In other words, with the same RSS threshold, the value of $r_{t h}$ will be higher in a 16 anchor scenario than in a 24 anchor scenario. Each group $S_{i}$ will have more nodes cooperating (in a 16 anchor scenario), hence the information is more reliable. It is also observed in Figure 6 that the threshold derived in the previous section $\left(R S S_{t h}^{P L}\right)$ has a similar behavior, interms of error, than the actual one obtained by means of simulation $\left(R S S_{t h}^{s i m}\right)$.

Our second issue is the reduction of the energy consumption. It is shown in Figure 7 that with a fixed number of $N_{m}$ the energy consumption is always higher when the network has a lower anchor density. As commented before, when the anchor node density is higher we need a higher value of the RSS threshold to achieve a certain value of $N_{m}$. That means a lower number of non-located nodes inside group $S_{i}$. In terms of efficient energy, then, it is better to deploy more anchor nodes in the network. However, this increases the cost of the

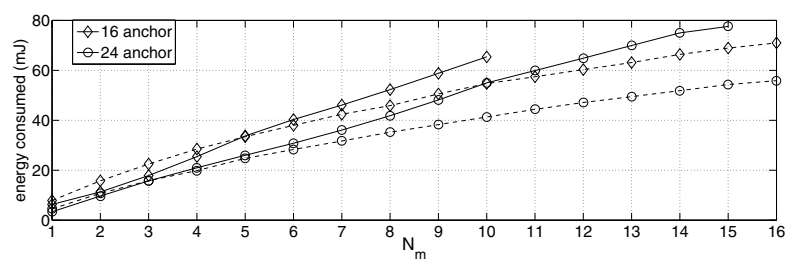

Fig. 7. Energy consumed versus mean number of anchor nodes(solid line: $R S S_{\text {th }}^{\text {sim }}$, dashed line: $R S S_{t h}^{P L}$ )

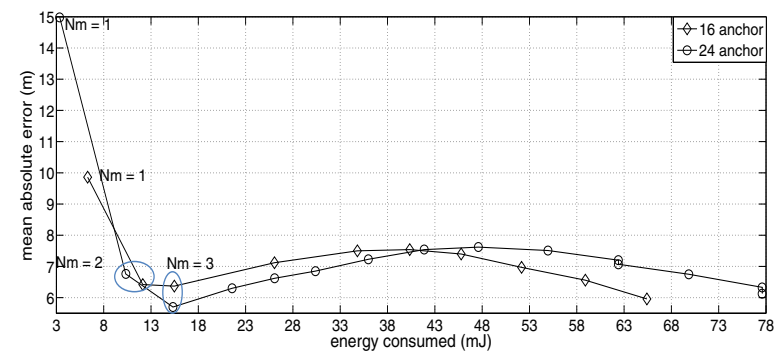

Fig. 8. Mean absolute error versus energy consumed

whole WSN.

As commented above, our interest is to optimize the position accuracy versus energy efficient tradeoff. For that reason, we present in Figure 8 the mean absolute error in terms of the energy consumption. As observed, the optimum point is at $N_{m}=3$ for both proposed scenarios. In a 24 anchor scenario we obtain an error of 5.9 meters and an energy consumption of $15.3 \mathrm{~mJ}$. On the other hand, in a 16 anchor node scenario, the error achieved is $6.4 \mathrm{~m}$ and an energy consumption of $15.5 \mathrm{~mJ}$. Comparing this consumption with the one obtained at the maximum radio range, the reduction achieved is an $80 \%$ and a $76 \%$ for a 24 and 16 anchor scenario, respectively.

In summary, it is observed that the proposed node selection technique is able to provide a good trade-off in terms of positioning accuracy vs. energy consumption when parameter $\mathrm{Nm}$ is set to 3. Therefore, the proposed strategy is quite appropriate for WSN deployment: each sensor has only to cooperate with those nodes with a RSS above a threshold and this threshold can be easily computed at each node by means of the proposed closed-form expression.

\section{B. Comparison with $M L$}

Next we compare the previous results (obtained with NodeSelection-LeastSquares algorithm) with those provided by a positioning system adopting Maximum Likelihood (ML) estimation, method where all the nodes are used in the cooperation process. In particular we compare both distributed methods in terms of position accuracy and energy consumption (see Figure 9).

A similar behaviour is observed for both schemes in terms of positioning accuracy. The NS-LS algorithm outperforms the ML method when the scenario has more than 14 anchor nodes, otherwise, ML method has a better accuracy but performance is slightly improved. Besides, NS-LS algorithm 


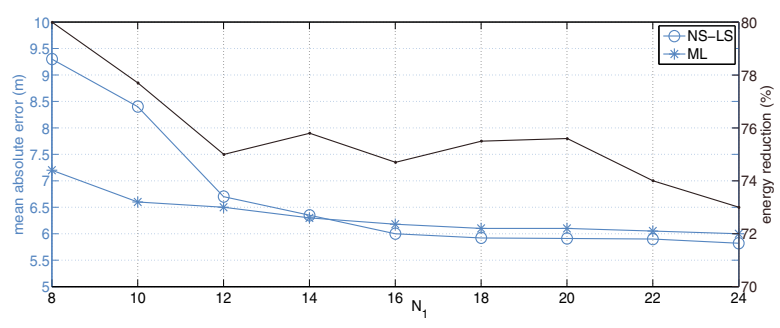

Fig. 9. Mean absolute error and percentage of reduction in energy consumption versus number of anchor nodes

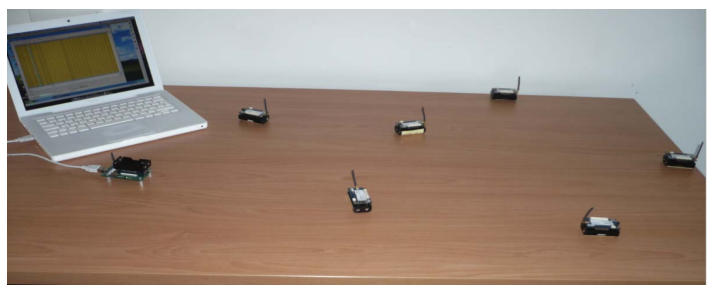

Fig. 10. IRIS Crossbow motes

provides approximately a $75 \%$ of reduction in terms of energy consumption with respect to ML.

\section{Experimental Validation}

In order to check the performance in a real scenario, we have done measurements in an indoor scenario with the IRIS motes @ $2.4 \mathrm{GHz}$ of Crossbow [10] (see Figure 10). The total number of nodes with unknow position, $N_{2}$, is 9 (see Figure 11). One can see in Figure 12 that in a real environment, a similar performance to that theoretically predicted in Figure 2 is achieved. By fixing a value of $N_{m}=3$ (as determined in subsection IV-A, the threshold is $-72 \mathrm{dBm}$ resulting in a position error equal to 1.25 meters. We can see in Figure 12, that the result obtained is near a local minimum of the error. Although this point is not the global minimum, the trade-off between energy and accuracy is good as energy is a increasing function of the RSS threshold.

Finally, it is worth noting that the values of the error obtained here are lower than the values achieved in previous computer simulations. This is justified by the fact that the experimental results are done for a small scale network.

\section{CONCLUSiON}

In this paper we have proposed a node selection criterion aimed at optimizing the position accuracy versus energy efficiency trade-off. Since wireless sensor networks are energy resource-constrained networks, our selection criterion fixes the RSS threshold, so it does not imply any additional cost in size or complexity. This threshold is computed to guarantee a mean number of anchor nodes $\left(N_{m}\right)$ inside the range of all the nodes of the network. The idea is to obtain an $N_{m}$ value that allows the optimization of the existing trade-off in terms of accuracy vs. energy consumption. An excellent behavior in terms of position accuracy versus energy trade-off is obtained with $N_{m}=3$. Besides, we have presented experimental results

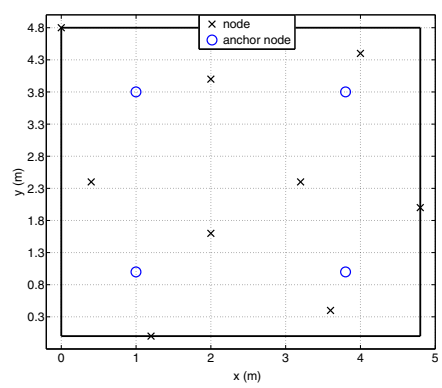

Fig. 11. Scenario deployed

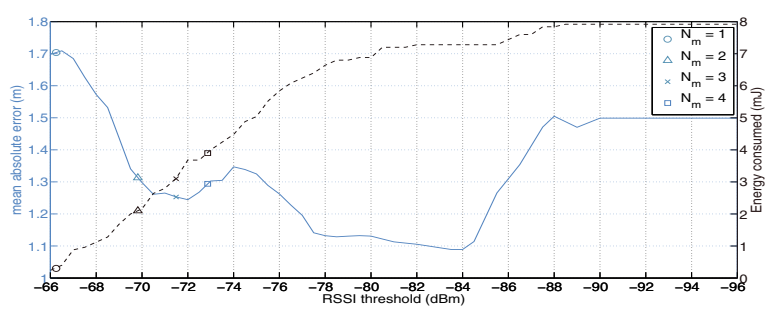

Fig. 12. Results of the mean absolute error and energy consumption vs. RSS threshold in a real indoor scenario (solid line: mean absolute error, dashed line: energy consumed)

that validates the proposed method and we have compared it with ML showing the efficiency of the solution. Hence, Node Selection-Least squares algorithm achieves a similar performance in terms of accuracy but an important reduction in terms of energy consumption is provided.

\section{REFERENCES}

[1] J. Bachrach and C. Taylor, Localization in Sensor Networks. Wiley and Sons, 2005.

[2] H. Wymeersch, J. Lien, and M. Win, "Cooperative localization in wireless networks," Proceedings of the IEEE, vol. 97, no. 2, pp. 427450, Feb. 2009.

[3] N. Patwari, J. Ash, S. Kyperountas, I. Hero, A.O., R. Moses, and N. Correal, "Locating the nodes: cooperative localization in wireless sensor networks," Signal Processing Magazine, IEEE, vol. 22, no. 4, pp. 54-69, July 2005.

[4] A. Boukerche, H. Oliveira, E. Nakamura, and A. Loureiro, "Localization systems for wireless sensor networks," Wireless Communications, IEEE, vol. 14, no. 6, pp. 6-12, December 2007.

[5] G. Shen, R. Zetik, and R. Thoma, "Performance comparison of toa and tdoa based location estimation algorithms in los environment," in Positioning, Navigation and Communication, 2008. WPNC 2008. 5th Workshop on, March 2008, pp. 71-78.

[6] D. Niculescu and B. Nath, "Ad hoc positioning system (aps) using aoa," in INFOCOM 2003. Twenty-Second Annual Joint Conference of the IEEE Computer and Communications. IEEE Societies, vol. 3, March-3 April 2003, pp. 1734-1743 vol.3.

[7] X. Li, "Collaborative localization with received-signal strength in wireless sensor networks," Vehicular Technology, IEEE Transactions on, vol. 56, no. 6, pp. 3807-3817, Nov. 2007.

[8] J. Ash and R. Moses, "On optimal anchor node placement in sensor localization by optimization of subspace principal angles," in Acoustics, Speech and Signal Processing, 2008. ICASSP 2008. IEEE International Conference on, 31 2008-April 4 2008, pp. 2289-2292.

[9] Y. Zou and K. Chakrabarty, "Energy-aware target localization in wireless sensor networks," in Pervasive Computing and Communications, 2003. (PerCom 2003). Proceedings of the First IEEE International Conference on, March 2003, pp. 60-67.

[10] Crossbow technology. [Online]. Available: www.xbow.com 